\title{
Machine Learning in Medicine: It Has Arrived, Let's Embrace it
}

\author{
scott Pappada ${ }^{1}$ \\ ${ }^{1}$ University of Toledo College of Medicine
}

September 24, 2021

\begin{abstract}
Machine learning and artificial intelligence (AI) in medicine has arrived in medicine and the healthcare community is experiencing significant growth in its adoption across numerous patient care settings. There are countless applications for machine learning and $\mathrm{AI}$ in medicine ranging from patient outcome prediction, to clinical decision support, to predicting future patient therapeutic setpoints. This commentary discusses a recent application leveraging machine learning to predict one year patient survival following orthotopic heart transplantation. This modeling approach has significant implications in terms of improving clinical decision making, patient counseling, and ultimately organ allocation and has been shown to significantly outperform preexisting algorithms. This commentary also discusses how adoption and advancement of this modeling approach in the future can provide increased personalization of patient care. The continued expansion of information systems and growth of electronic patient data sources in healthcare will continue to pave the way for increased use and adoption of data science in medicine. Personalized medicine has been a long-standing goal of the healthcare community and with machine learning and AI now being continually incorporated into clinical settings and practice, this technology is well on the pathway to make a considerable impact to greatly improve patient care in the near future.
\end{abstract}

Machine Learning in Medicine: It Has Arrived, Let's Embrace it

Scott M Pappada, $\mathrm{PhD}^{1,2,3,4}$

${ }^{1}$ Department of Anesthesiology, The University of Toledo College of Medicine, Toledo, OH 43614, USA

${ }^{2}$ Department of Bioengineering, The University of Toledo, Toledo, OH 43606, USA

${ }^{3}$ Department of Electrical Engineering and Computer Science, The University of Toledo, Toledo, OH 43606, USA

${ }^{4}$ Department of Anesthesiology, The Ohio State University Wexner Medical Center, Columbus, OH 43210, USA.

It is safe to say that machine learning and artificial intelligence (AI) in medicine has arrived and it is time for the healthcare community to embrace it. There has been significant recent growth in the adoption and use of machine learning in medicine, and researchers and healthcare professionals are trending towards leveraging it for multiple purposes including, for example, in the prediction of patient outcomes which are being used to drive decision-making surrounding patient interventions and care. An excellent recent demonstration of the adoption of machine learning in medicine is the recent manuscript by Ayers et al[1], whose team developed an ensemble machine learning-based model to predict patient survival one year following orthotopic heart transplantation (OHT). The primary purpose of this machine learning approach was outcome prediction (i.e. , likelihood of patient survival one year after organ transplantation), however, as the authors allude to, this modeling approach also has significant implications in terms of improving clinical decision making, patient counseling, and ultimately organ allocation. This particular modeling approach significantly outperforms other established OHT algorithms created by Nilsson et al. in 2015 and Weiss et al in 2011[2, 3 . The improved accuracy of the ensemble model developed by Ayers and colleagues with respect to these 
prior algorithms can perhaps be tied to the fact that its predictions are based on the output of hundreds of models, where each model's training data is generated by employing varying degrees of data re-sampling of the comprehensive United Network for Organ Sharing (UNOS) database which is growing in its use for "Big Data" applications by the healthcare community[4]. Ayers and colleagues therefore have effectively produced hundreds of models each of which were developed using diverse training data sets which will improve the capability of the models (400 in this case) to evaluate different relationships across the dataset. This effectively contributes to improvements in the prediction of patient survival one year following transplantation. The use of machine learning-based approaches, such as this ensemble modeling approach, provides a mechanism to evaluate a diverse set of variables $(\mathrm{N}=525)$ and factors far beyond traditional measures which drive organ allocation, that include but are not limited to the medical need of the recipient, compatibility of blood type, and physical proximity between recipient and the donor. Machine learning-based approaches are uniquely suited to evaluate hundreds of preoperative factors and variables that provides a more personalized organ allocation recommendation.

When designed and utilized appropriately, machine learning-based software and technologies will provide tremendous value across many clinical settings. Let's investigate the potential implications of incorporating the machine learning-based approach proposed by Ayers et al.[1] into clinical practice and its potential impact on patient care given future adoption and advancement of the models. As discussed, these machine learning algorithms provide potential to significantly improve not only organ allocation but a means to optimize patient longevity following transplantation. Ayers and colleagues provide an envisioned use case where the model is utilized iteratively each time an organ becomes available across several candidates to identify which patient has the highest likelihood of reaching the one-year survival milestone following transplantation. This data-driven process provides a more personalized recommendation of patient survivability and has the potential to evaluate numerous clinical, psychological, and behavioral measures that significantly surpass existing conventional selection criteria. With machine learning-based approaches and the continued growth in patient data availability, many other data sources, factors, and variables can be investigated and incorporated into other models that may arise and are adopted in the future. Increased availability of salient patient data will result in a significant improvement in model performance and accuracy over time. An example of improved patient data availability would be data collected via ambulatory patient monitoring, both postoperatively and post hospital admission. Ambulatory patient monitoring is becoming more commonplace and routine especially with the increased adoption of telemedicine[5, 6]. Wearable devices such as the FitBit ${ }^{\circledR}$ which are used across the general population are now beginning to be widely investigated for use across patient populations. [7, 8] These wearable devices offer a unique potential to document factors that can be incorporated as model inputs/predictors to improve model-generated recommendations for organ allocation, patient counseling, and many other aspects[9-11]. In this future use case, healthcare providers can keep track of important factors such as patient activity[12], emotional status, and sleep quality[13, 14] both preoperatively (for organ allocation purposes) and postoperatively (to improve patient compliance and ultimately survivability). The adoption of machine learning coupled with current standard organ allocation criteria provides a unique potential to not only optimize organ allocation but to provide better insight into patient care requirements after a successful transplantation. As more of these technologies are utilized across larger patient groups, the additional data collected can be utilized in a dynamic sense to continually enhance the real-time performance of existing models and to further contribute to improvements in model accuracy. This same approach could also be applicable in patient survival models where the additional data collected during each patient encounter will continuously enhance model performance. Healthcare providers will be able to evaluate predictions and recommend changes to patient treatment, lifestyle, or behaviors that may improve upon survivability. Adding additional machine learning algorithms such as unsupervised techniques may also allude to patterns amongst specific patient cohorts and support the identification of various factors that may contribute to positive or negative patient outcomes. This will effectively allow healthcare providers to deliver more focused and personalized patient care.

The increased adoption of advanced patient monitoring technologies coupled with the expansion and advancement of electronic medical records (EMR) continues to generate significant sets of data across numerous 
patient care settings. Along with this considerable growth in electronic data, the cognitive and physical burden of healthcare providers (HCPs) to interpret these patient data sources also continues to grow. To ensure optimal patient care, providers must adapt to this continuously evolving space and evaluate the output of these new technologies and data sources when arriving at their patient care and treatment decisions. The persistent growth of medical technologies and electronic healthcare data has shepherded medicine into the era of "Big Data"[15]. While the need to interpret and apply knowledge from these various patient data sources can sometimes be overwhelming for $\mathrm{HCPs}[16]$, the availability of significant datasets provides a critical foundation for future use by the medical research community to assist in developing innovative healthcare technology solutions through the use of machine learning and AI. Consistent with this trend, the United States Food and Drug Administration (FDA) realizes that machine learning and AI hold tremendous promise for the future of medicine, and as a result are developing a new regulatory framework to promote innovation in this space and to support the use of AI-based technologies[17]. Digital health tools which use machine learning and AI have significant potential to improve the abilities of HCPs in the diagnosis, treatment, decision making, and even healthcare administration. These data driven tools, algorithms, and techniques offers the unique potential for medical care to become truly personalized at the patient level. There are countless applications for data science in healthcare. One application includes the diagnosis of key medical conditions and disease states including but not limited to cancer[18], sepsis/septic shock[19, 20], and developmental disabilities such as autism spectrum disorder[21, 22]. Machine learning has also been applied to drive clinical decision making and to provide decision support in a diverse set of clinical settings and specialties including but not limited to neurosurgery[23], cardiovascular medicine/cardiac surgery[24, 25], endocrinology[26-28], radiology[29], and critical care[30-32]. It has also been leveraged for time series prediction of specific patient vital signs [33, 34] or even therapeutic setpoints[28, 31, 35]. Similarly, algorithms and modeling approaches have also been applied to prediction of patient outcomes including mortality[36, 37], length of stay in the hospital[38, 39] or intensive care unit[40, 41], readmission[42-44], and countless others.

In medicine today, optimal patient care, including the delivery of treatment to improve patient outcomes, relies on the rigorous monitoring of a large quantity of patient data which often can be distributed across various information systems. The ability to properly identify, track, and interpret each related data source can be time consuming, labor intensive, and will result in cognitive overload for even the most experienced of healthcare professionals. Therefore, machine learning and AI in medicine will definitely play a significant role in positively impacting patient safety and care due to its ability to better manage and interpret "big-data" sources. As has been shown, when software and technologies implementing machine learning are designed appropriately, they are able to significantly reduce experienced cognitive load[45] of healthcare providers, thereby, providing a better working environment and potentially leading to reductions in provider burnout[4648] which is a major issue facing our healthcare workforce today. Furthermore, staff and resources are often constrained in healthcare environments and useful data, trends, and/or correlations between variables often go unnoticed because healthcare professionals do not have the time or capacity to properly evaluate all incoming data. This is where software tools and technologies that implement machine learning and AI can be of tremendous value. While many factors and variables are documented in patient medical records, they aren't necessarily incorporated into the decision-making process. Data science can be leveraged to identify subtle patterns or factors that are of specific interest and allow for a more effective prediction of patient outcomes of interest. It is now possible to find patterns and trends amongst seemingly unrelated data sources which may not even be part of routine clinical evaluations and/or assessment practices. While machine learning has considerable potential, it should be stressed that it alone cannot eliminate uncertainty in clinical care. It can, however, provide a means to rapidly evaluate a multitude of variables and factors that would take even an experienced provider a significant amount of time to process and draw conclusions. This is one of its greatest advantages. The "Big Data Revolution" has arrived and machine learning and AI in medicine is here to stay. Software and patient monitoring technologies implementing sophisticated models and algorithms will soon be commonplace across healthcare settings. While being a promoter of machine learning and AI in healthcare, it is also important to note that they will never replace a seasoned and experienced healthcare provider. They will, however, provide healthcare providers an extremely valuable tool to guide and augment their expert clinical decision-making. Personalized medicine has been a long-standing goal of the healthcare 
community and with machine learning and AI now being continually incorporated into clinical settings and practice, this technology is well on the pathway to make a considerable impact to greatly improve patient care in the near future.

\section{References Cited}

1. Ayers B, Sandholm T, Gosev I, Prasad S, Kilic A, Using Machine Learning to Improve Survival Prediction After Heart Transplantation Surgery. Journal Of Cardiac Surgery, 2021.

2. Nilsson, J., et al., The International Heart Transplant Survival Algorithm (IHTSA): A New Model to Improve Organ Sharing and Survival. PLOS ONE, 2015. 10 (3): p. e0118644.

3. Weiss, E.S., et al., Creation of a quantitative recipient risk index for mortality prediction after cardiac transplantation (IMPACT).The Annals of thoracic surgery, 2011. 92 (3): p. 914-922.

4. Massie, A.B., L.M. Kucirka, and D.L. Segev, Big data in organ transplantation: registries and administrative claims. American journal of transplantation : official journal of the American Society of Transplantation and the American Society of Transplant Surgeons, 2014.14 (8): p. 1723-1730.

5. Hung, K. and Y.-T. Zhang, Implementation of a WAP-based telemedicine system for patient monitoring. IEEE transactions on Information Technology in Biomedicine, 2003. 7 (2): p. 101-107.

6. Koehler, F., et al., Telemedical Interventional Monitoring in Heart Failure (TIM-HF), a randomized, controlled intervention trial investigating the impact of telemedicine on mortality in ambulatory patients with heart failure: study design. European journal of heart failure, 2010. 12 (12): p. 1354-1362.

7. Low, C.A., et al., Fitbit step counts during inpatient recovery from cancer surgery as a predictor of readmission. Annals of Behavioral Medicine, 2018. 52 (1): p. 88-92.

8. Daligadu, J., et al., Validation of the Fitbit Flex in an acute post-cardiac surgery patient Population. Physiotherapy Canada, 2018.70 (4): p. 314-320.

9. Macapagal, F.R. and H.M. Rodriguez, CICU PreHeart Transplant 1A Patients Experiences Using Fitbit Activity Tracker in Their Own Words.

10. Macapagal, F.R., et al., Experiences of Pre-Heart Transplant Patients Using Fitbit as an Ambulation Measuring Device.

11. Macapagal, F.R., et al., Use of common technology (Fitbit) as an aid to increase ambulation among pre-heart transplant patients.

12. Hartman, S.J., S.H. Nelson, and L.S. Weiner, Patterns of Fitbit use and activity levels throughout a physical activity intervention: exploratory analysis from a randomized controlled trial.JMIR mHealth and uHealth, 2018. 6 (2): p. e8503.

13. Liao, Y., et al., Investigating the within-person relationships between activity levels and sleep duration using Fitbit data. Translational behavioral medicine, 2021. 11 (2): p. 619-624.

14. Rezaei, N. and M.A. Grandner, Changes in sleep duration, timing, and variability during the COVID-19 pandemic: Large-scale Fitbit data from 6 major US cities. Sleep Health, 2021.

15. Austin, C. and F. Kusumoto, The application of Big Data in medicine: current implications and future directions. Journal of Interventional Cardiac Electrophysiology, 2016. 47 (1): p. 51-59.

16. Holden, R.J., Cognitive performance-altering effects of electronic medical records: an application of the human factors paradigm for patient safety. Cognition, Technology \& Work, 2011.13 (1): p. 11-29.

17. Administration, U.F.A.D. Transforming FDA's Approach to Digital Health . 2018 [cited July 2018; Available from: https://www.fda.gov/newsevents/speeches/ucm605697.htm. 
18. Manogaran, G., et al., Machine learning based big data processing framework for cancer diagnosis using hidden Markov model and GM clustering. Wireless personal communications, 2018. 102 (3): p. 2099-2116.

19. Burdick, H., et al., Validation of a machine learning algorithm for early severe sepsis prediction: a retrospective study predicting severe sepsis up to $48 \mathrm{~h}$ in advance using a diverse dataset from 461 US hospitals. BMC medical informatics and decision making, 2020. 20 (1): p. 1-10.

20. Fagerstrom, J., et al., LiSep LSTM: a machine learning algorithm for early detection of septic shock. Scientific reports, 2019. 9 (1): p. 1-8.

21. Parikh, M.N., H. Li, and L. He, Enhancing diagnosis of autism with optimized machine learning models and personal characteristic data. Frontiers in computational neuroscience, 2019. 13 : p. 9.

22. Tariq, Q., et al., Mobile detection of autism through machine learning on home video: A development and prospective validation study.PLoS medicine, 2018. 15 (11): p. e1002705.

23. Buchlak, Q.D., et al., Machine learning applications to clinical decision support in neurosurgery: an artificial intelligence augmented systematic review. Neurosurgical review, 2020.43 (5): p. 1235-1253.

24. Kilic, A., Artificial intelligence and machine learning in cardiovascular health care. The Annals of thoracic surgery, 2020.109 (5): p. 1323-1329.

25. Tseng, P.-Y., et al., Prediction of the development of acute kidney injury following cardiac surgery by machine learning. Critical Care, 2020. 24 (1): p. 1-13.

26. Tyler, N.S., et al., An artificial intelligence decision support system for the management of type 1 diabetes. Nature metabolism, 2020. 2 (7): p. 612-619.

27. Pappada, S.M., B.D. Cameron, and P.M. Rosman, Development of a neural network for prediction of glucose concentration in type 1 diabetes patients. Journal of diabetes science and technology, 2008.2 (5): p. 792-801.

28. Pappada, S.M., et al., Neural network-based real-time prediction of glucose in patients with insulindependent diabetes.Diabetes technology \& therapeutics, 2011. 13 (2): p. 135-141.

29. Syeda-Mahmood, T., Role of big data and machine learning in diagnostic decision support in radiology. Journal of the American College of Radiology, 2018. 15 (3): p. 569-576.

30. Pirracchio, R., et al., Big data and targeted machine learning in action to assist medical decision in the ICU. Anaesthesia Critical Care \& Pain Medicine, 2019. 38 (4): p. 377-384.

31. Pappada, S.M., et al., An artificial neural network-based predictive model to support optimization of inpatient glycemic control.Diabetes technology \& therapeutics, 2020. 22 (5): p. 383-394.

32. Pappada, S.M., et al., Evaluation of a model for glycemic prediction in critically ill surgical patients. PLoS One, 2013.8 (7): p. e69475.

33. Youssef Ali Amer, A., et al., Vital signs prediction and early warning score calculation based on continuous monitoring of hospitalised patients using wearable technology. Sensors, 2020. 20 (22): p. 6593.

34. Zhang, B., et al., Health data driven on continuous blood pressure prediction based on gradient boosting decision tree algorithm.IEEE Access, 2019. 7 : p. 32423-32433.

35. Rodriguez-Rodriguez, I., et al., Utility of big data in predicting short-term blood glucose levels in type 1 diabetes mellitus through machine learning techniques. Sensors, 2019. 19 (20): p. 4482.

36. Raj, R., et al., Machine learning-based dynamic mortality prediction after traumatic brain injury. Scientific reports, 2019.9 (1): p. 1-13.

37. Gao, Y., et al., Machine learning based early warning system enables accurate mortality risk prediction for COVID-19. Nature communications, 2020. 11 (1): p. 1-10. 
38. Daghistani, T.A., et al., Predictors of in-hospital length of stay among cardiac patients: A machine learning approach. International journal of cardiology, 2019. 288 : p. 140-147.

39. Thompson, B., K.O. Elish, and R. Steele. Machine learning-based prediction of prolonged length of stay in newborns . in2018 17th IEEE International Conference on Machine Learning and Applications (ICMLA) . 2018. IEEE.

40. Alsinglawi, B., et al. Predicting Length of Stay for Cardiovascular Hospitalizations in the Intensive Care Unit: Machine Learning Approach . in 2020 42nd Annual International Conference of the IEEE Engineering in Medicine 85 Biology Society (EMBC) . 2020. IEEE.

41. Ling, Y., et al., A prediction model for length of stay in the ICU among septic patients: A machine learning approach. Value in Health, 2018. 21 : p. S5.

42. Morgan, D.J., et al., Assessment of machine learning vs standard prediction rules for predicting hospital readmissions. JAMA network open, 2019. 2 (3): p. e190348-e190348.

43. Rojas, J.C., et al., Predicting intensive care unit readmission with machine learning using electronic health record data.Annals of the American Thoracic Society, 2018. 15 (7): p. 846-853.

44. Jiang, S., et al., An integrated machine learning framework for hospital readmission prediction. Knowledge-Based Systems, 2018.146 : p. 73-90.

45. Miller, K., et al., Interface, information, interaction: a narrative review of design and functional requirements for clinical decision support. Journal of the American Medical Informatics Association, 2018. 25 (5): p. $585-592$.

46. Bakken, S., Can informatics innovation help mitigate clinician burnout? 2019, Oxford University Press.

47. Mahadevaiah, G., et al., Artificial intelligence-based clinical decision support in modern medical physics: selection, acceptance, commissioning, and quality assurance. Medical physics, 2020. 47 (5): p. e228-e235.

48. Hunt, D.L., et al., Effects of computer-based clinical decision support systems on physician performance and patient outcomes: a systematic review. Jama, 1998. 280 (15): p. 1339-1346. 